\title{
Study on Influencing Factor Analysis and Application of Consumer Mobile Commerce Acceptance
}

\author{
Gaoguang $\mathrm{Li}^{1}$ and Tingjie $\mathrm{Lv}^{2}$ \\ 1 School of Economics and Management, Beijing University of Posts and \\ Telecommunications, Beijing 100876, P.R. China,ligaoguang@gmail.com \\ 2 School of Economics and Management, Beijing University of Posts and \\ Telecommunications, Beijing 100876, P.R. China, lutingjie@263.net
}

\begin{abstract}
Mobile commerce (MC) refers to e-commerce activities carried out using a mobile device such as a phone or PDA. With new technology, MC will be rapidly growing in the near future. At the present time, what factors making consumer accept $\mathrm{MC}$ and what $\mathrm{MC}$ applications are acceptable by consumers are two of hot issues both for $\mathrm{MC}$ providers and $\mathrm{f}$ or $\mathrm{MC}$ researchers. This study presents a proposed MC acceptance model that integrates perceived playfulness, perceived risk and cost into the TAM to study which factors affect consumer $\mathrm{MC}$ acceptance. The proposed model includes five variables, namely perceived risk, cost, perceived usefulness, perceived playfulness, perceived ease of use, perceived playfulness. Then, using analytic hierarchy process (AHP) to calculate weight of criteria involved in proposed model. Finally, the study utilizes fuzzy comprehensive evaluation method to evaluate MC applications accepted possibility, and then a MC application is empirically tested using data collected from a survey of MC consumers.
\end{abstract}

\section{Introduction}

Early studies on mobile commerce (MC) suggest that there is a general consumer interest toward $\mathrm{MC}$ and service applications. Purchases on web sites, electronic receipts and tickets, mobile content, routine bank services, peer-to-peer payments, and vending are among the potential applications [1]. However, the adoption of MC and services has been slower than expected. Facing on situation of insufficient user acceptance, Different persons have given different explanation from diverse angles. For promoting healthy development of $\mathrm{MC}$, we believe that it is extremely important to understand consumer $\mathrm{MC}$ perceptions and acceptance. We believe that there are

Please use the following format when citing this chapter:

I.i, G., Iv, T., 2007, in IFIP International lederation for Information Processing, Volume 252, Integration and Innovation Orient to F-Society Volume 2, eds. Wang, W., (Boston: Springer), pp. 132-141. 
some important issues (i.e., cost, risk, usefulness, ease of use, playfulness) that can explain why some mobile applications have been successful and why some others have not performed as expected.

In this study we aim to analyze which factors will affect and how to affect consumer adoption to $\mathrm{MC}$ and analyze accepted possibility of certain $\mathrm{MC}$ application. The theoretical background of the study is based on the technology acceptance model (TAM) and diffusion of innovations theory, which have been suggested by prior studies as applicable frame of reference for MC context. Cited empirical data in the study was obtained from survey to some specialists in the relative field.

The paper is organized as follows. In the next section, we discuss the ingredients of affecting consumer adoption to $\mathrm{MC}$ in virtue of TAM and production of other research fields, we then construct a research model for $\mathrm{MC}$ acceptance and build an index system for evaluating. In the third section we apply the analytic hierarchy process (AHP) to evaluation of weight of factors in upper model. In succession, choosing a piece of $\mathrm{MC}$ applications, we use fuzzy evaluating method to analyze the accepted possibility by consumers. The final chapter discusses our results and suggests future research.

\section{Research model}

\subsection{Perceived usefulness and perceived ease of use}

The TAM is an information systems theory that models how users come to accept and use a technology. The TAM was first introduced by Davis et al. in 1986 [2].TAM is a well respected model of IT adoption and use. The model provides a traditional view point about technology acceptance from the users' perspective. The model provides a traditional view point about technology acceptance from the users' perspective.

The core concept of TAM is that perceptions of usefulness, ease of use and other external variables will influence an individual's intention to use IT, which will ultimately influence actual usage behavior [2]. Perceived usefulness and perceived ease of use are the two particular beliefs. According to the model, system usage is determined by the users' attitude towards using the system while attitude towards using is jointly determined by usefulness and ease of use [2]. Perceived usefulness is defined as "the degree to which a person believes that using a particular system would enhance performance".

Perceived ease of use is defined as "the degree to which a person believes that using a particular system would be free of physical and mental efforts" [2]. Davis also suggested that perceived ease of use may actually be a prime causal antecedent of perceived usefulness. TAM also postulates that perceived ease of use is an important determinant of attitude toward using a system. 


\subsection{Cost and perceived risk}

Much research also indicated that TAM needed integration with additional variables in order to improve its prediction of system use [3]. Chen and Hitt et al pointed out consumers must deal with non-negligible costs in switching between different brands of products or relative services in various markets. Transitioning from wired EC to $\mathrm{MC}$ implies some additional expenses. Equipment costs, access cost, and transaction fees are three important components that make MC use more expensive than wired EC. In addition, frustrating experiences, such as slow connections, poor quality, outof-date content, missing links, and errors have infuriated online users. Unfortunately, consumers must pay for all these frustrations [4]. Some researchers suggested that $\mathrm{MC}$ providers should find solutions that reduce the costs and entice present and new customers to access portals anytime, from anywhere.

According to cost-efficiency model, cost is important factors in consumer decision-making of purchase and use. Therefore, the perceived cost is an important factor affecting. It has a negative direct effect on behavioral intention to use. With the increasingly high penetration rate of Internet applications, people are anxious about the diverse types of risks presented when engaging in online activities or transactions. When customers are uncertain about product quality, brands and online services they may worry about an unjustifiable delay in product delivery, providing payment without receiving the product and other illegal activities and fraud. Perceived risk refers to certain types of financial, product performance, social, psychological, physical, or time risks when consumers make transactions online

Credit ratings, bank balances and financial data could be changed without the owner knowing during online transactions. The reliability of online transactions is still far from perfect. Cognitive and affective factors are important variables that prevent people from trusting online services. Pavlou defines this as "the user's subjective expectation of suffering a loss in pursuit of a desired outcome" [5]. Other research also indicated that perceived risk is an important determinant of consumers' attitude toward online transactions. Since intention to use a website for transactions involves a certain degree of uncertainty, perceived risk is incorporated as a direct antecedent of behavioral intention to use. Hence, Perceived risk has a negative direct effect on behavioral intention to use [4].

\subsection{Perceived playfulness}

For nailing down consumer's intrinsic motivation to computers acceptance, Davis et al introduce into the conception of perceived playfulness. Perceived playfulness is defined as feeling playful and dispensing with thinking over evocable aftereffect possibly. In theory, perceived playfulness affects directly use intention. In American, there were two research carried out to study affection of playfulness. One was the study of 200 American MBA students using word-processing procedure, the other was the research on 40 American MBA students using graphical procedure. The results were showed as follows: there are stronger relationship between usefulness and use intention, at the same time, perceived playfulness and use intention has 
weaker relationship, perceived ease of use affect actual use through perceived usefulness and perceived playfulness.

Early empirical research on use of Internet shows that perceived playfulness is the main driving force of consumer using Internet. For example, Atkinson and Kydd (1997) validate the relation between use of Internet, perceived usefulness and perceived pleasure through survey on $84 \mathrm{MBA}$ students and 78 masters major in business administration. They find that perceived playfulness has an obvious effect on use of Internet but perceived usefulness is not very obvious when consumer uses Internet for entertainment. They find that there are stronger relationship between perceived usefulness and actual use but weaker relationship between perceived playfulness and actual use when consumer uses Internet for study [6].

Teo et al (1999) research how three factors (i.e., perceived ease of use, perceived usefulness, perceived playfulness) affect consumer use of Internet by means of online questionnaire. Analyzing 1370 questionnaires obtained, they find that Internet users are largely impact by perceived usefulness and perceived playfulness. However, Kydd and Atkinson draw an opposite conclusion, they find that perceived usefulness has a weaker effect on frequent user and perceived has also a weaker effect on online frequency. According to questionnaires of 152 college students in South Korea, Moon and Kim find perceived usefulness and playfulness have an obvious effect on consumer use of Internet. Hence, we believe perceived playfulness has a positive effect on behavioral intention to use.

\subsection{Proposed research model}

The research model for our study is depicted in figure 1 . In the model, based on these arguments, our study integrates TAM and three additional variables (i.e., cost, perceived risk, and perceived playfulness) to model user acceptance in the $\mathrm{B} 2 \mathrm{C} \mathrm{MC}$ context. The constructs of perceived ease of use, perceived usefulness, behavioral intention to use, and actual use are adopted from TAM. Three other external variables are also integrated into the model.

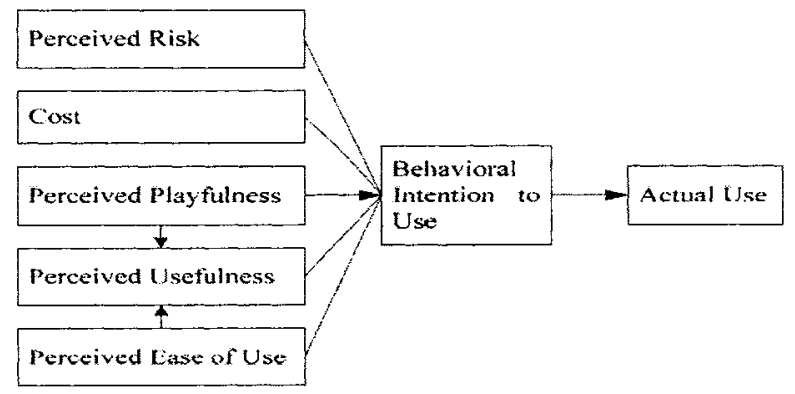

Fig. 1. Proposed MC acceptance model 


\subsection{Construct criteria system of MC application acceptance}

Many variables involved in social, scientific, psychological research can't be measured directly, this variable is named as latent variable, so we measure indirectly latent variable by observable indicators. In the study, there are seven latent variables, namely perceived usefulness, perceived ease of use, behavior intention to use, actual use, perceived playfulness, perceived risk and cost. Observable indicators refer to previous research and actual situation. Previous research was reviewed to ensure that a comprehensive list of measures were included. Those for perceived usefulness, perceived ease of use, behavioral intention to use and actual use were adapted in our model from previous studies on TAM (e.g., [7]). The construct for perceived risk was adapted from the studies of Pavlou and Eastin. The scales for playfulness were based on M. Igbaria et al. and Atkinson et al. The measures for cost were captured using three items derived from Constantinides, Rupp and Smith, and real world experience. For perceived ease of use has slim effect, we omit it. After a series of modifying and adjusting, the evaluating criteria system is depicted in figure 2 .

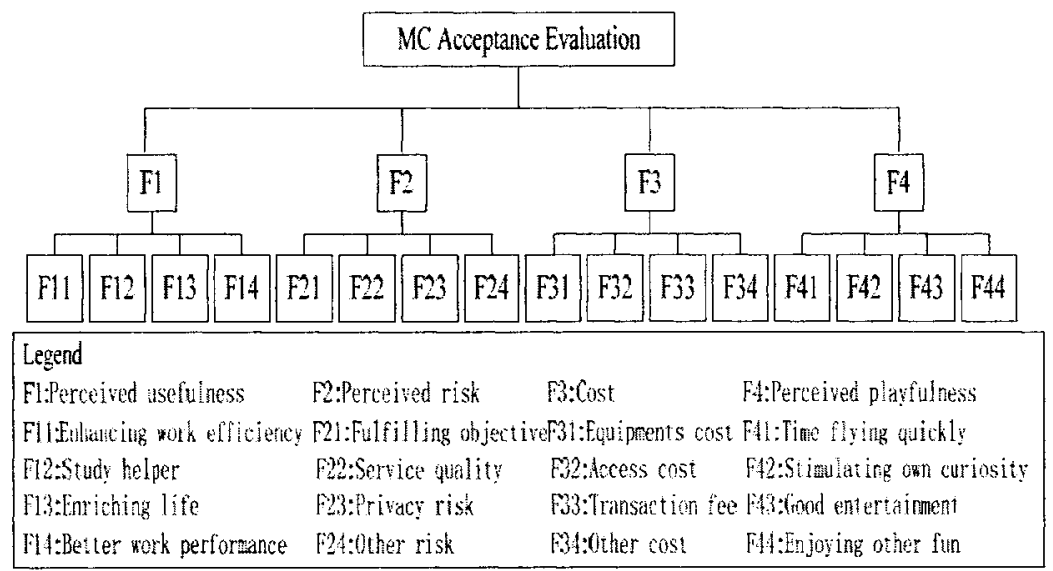

Fig. 2. Criteria system of $m c$ application acceptance \& AHP hierarchical diagram

\section{Evaluating weight of criteria through AHP}

AHP is a multi-criteria decision method that utilizes structured pair-wise comparisons among systems of similar alternative strategies to produce a scale of preference. It has been found to be an effective approach that can settle complex decision. And it has been used by numerous researchers in various fields to handle both tangible and intangible factors and sub-factors. As the AHP approach is a subjective methodology, information and the priority weighs of elements may be obtained from a decision-maker using direct questioning or by a questionnaire method. The AHP procedure is usually summarized as four steps, now we will 
evaluate weight of factor step by step and prepare for using fuzzy comprehensive evaluation to evaluate possibility of certain MC application acceptance.

\subsection{Establish hierarchy structure (Step 1)}

In this step, we build a decision hierarchy by breaking a general problem into individual criteria. Figure. 2 in section 2 is the AHP hierarchical diagram. The top of the hierarchy is the overall objective, the decision alternatives are at the bottom. The middle nodes are the relevant attributes (criteria) of the decision problem.

\subsection{Construct judgment matrix (Step 2)}

Next, we gather rational data for the decision criteria and alternatives, using the AHP relational scale suggested by T. L. Saaty. We invite five specialists to carry out pairwise comparison of elements at each level in the hierarchy. From now on, we take example for perceived usefulness, and other results can obtain in terms of same method. Table 1 show the original pair-wise weighs of the AHP matrices.

Table 1

\begin{tabular}{ccccc}
$\mathrm{C}$ & $\mathrm{u}_{1}$ & $\mathrm{u}_{2}$ & $\mathrm{u}_{3}$ & $\mathrm{u}_{4}$ \\
\hline $\mathrm{u}_{1}$ & 1 & $1 / 2$ & 2 & 3 \\
$\mathfrak{u}_{2}$ & 2 & 1 & 2 & 2 \\
$\mathrm{u}_{3}$ & $1 / 2$ & $1 / 2$ & 1 & 2 \\
$\mathrm{u}_{4}$ & $1 / 3$ & $1 / 2$ & $1 / 2$ & 1
\end{tabular}

\subsection{Obtain weights of criteria}

The relative weights of the criterion of each level with respect to an element in the adjacent upper level are computed as the components of the normalized eigenvector associated with the largest eigenvalue of their comparison matrix. The composite weights of the decision alternative are then determined by aggregating the weights through the hierarchy. Now we will make calculation to judgment matrix and obtain weights of various evaluating criterion.

Firstly, using the following formula:

$$
\begin{gathered}
W_{i}=\frac{1}{n} \sum_{j=1}^{n} \frac{c_{i j}}{\sum_{\substack{k=1 \\
\text { to }}}^{n} c_{k j}} i=1,2, \cdots, n \\
\text { According in Tab } \\
\text { vector. } W=\left(\begin{array}{llll}
0.299 & 0.384 & 0.191 & 0.126
\end{array}\right)
\end{gathered}
$$

\subsection{Consistency test}

Constructing judgment matrix should keep thought consistency, only when matrix is consistent totally, there exists $\lambda_{\max }=n$ in judgment matrix, however, 
there exists $\lambda_{\max }>n$. Hence, the difference between $\lambda_{\max }$ and $n$ is used to test consistency using $C I$. The relation between $C I$ and $\lambda_{\max }$ is described as follows:

$$
C I=\frac{\lambda_{\max }-n}{n-1}
$$

$\lambda_{\max }$ is matrix maximal latent root, and $n$ represents matrix rank, the smaller $C I$, the better consistency. At the same time, $C I$ is necessary to compare with random consistency index ( $R I)$, we can obtain the test value, namely $C R$, the formula and condition are as follows:

$$
C R=\frac{C I}{R I} \quad C R<0.1
$$

And then we verify consistency of judgment matrix by upper method.

$$
\begin{gathered}
C W=\left(\begin{array}{cccc}
1 & \frac{1}{2} & 2 & 3 \\
2 & 1 & 2 & 2 \\
\frac{1}{2} & \frac{1}{2} & 1 & 2 \\
\frac{1}{3} & \frac{1}{2} & \frac{1}{2} & 1
\end{array}\right)\left(\begin{array}{c}
0.299 \\
0.384 \\
0.191 \\
0.126
\end{array}\right)=\left(\begin{array}{c}
1.330 \\
1.616 \\
0.785 \\
0.513
\end{array}\right) \\
\lambda_{\max }=\sum_{i=1}^{n} \frac{(C W)_{i}}{n W}=4.209 \quad C I=\frac{\lambda_{\max }-n}{n-1}=0.0697 \quad C R=\frac{C I}{R I}=0.0774<0.10
\end{gathered}
$$

Therefore, compositor result of various criterion weight vectors is satisfied in

\begin{tabular}{|c|c|c|c|c|c|c|c|c|}
\hline $\begin{array}{l}\text { Criteria } \\
\text { (level 1) }\end{array}$ & weight & $\begin{array}{l}\text { Criteria } \\
\text { (level 2) }\end{array}$ & $\begin{array}{l}\text { Wei- } \\
\text { ght }\end{array}$ & $A$ & $B$ & $c$ & D & $E$ \\
\hline \multirow{4}{*}{$\begin{array}{l}\text { Perceived } \\
\text { Usefulness }\end{array}$} & \multirow[t]{4}{*}{0.272} & F11 & 0.299 & 4 & 0 & 4 & 11 & 1 \\
\hline & & F12 & 0.384 & 1 & 1 & 3 & 6 & 9 \\
\hline & & F13 & 0.191 & 0 & 0 & 8 & 12 & 0 \\
\hline & & F14 & 0.126 & 0 & 3 & 8 & 9 & 0 \\
\hline \multirow{4}{*}{$\begin{array}{l}\text { Perceived } \\
\text { Risk }\end{array}$} & \multirow[t]{4}{*}{0.319} & F21 & 0.281 & 0 & 4 & 6 & 8 & 2 \\
\hline & & F22 & 0.277 & 2 & 0 & 4 & 6 & 8 \\
\hline & & F23 & 0.231 & 2 & 2 & 4 & 4 & 8 \\
\hline & & $\mathrm{F} 24$ & 0.211 & 0 & 4 & 8 & 8 & 0 \\
\hline \multirow[t]{4}{*}{ Cost } & \multirow[t]{4}{*}{0.239} & F31 & 0.271 & 0 & 0 & 2 & 4 & 14 \\
\hline & & F32 & 0.256 & 8 & 2 & 2 & 6 & 2 \\
\hline & & F33 & 0.241 & 4 & 2 & 2 & 2 & 10 \\
\hline & & F34 & 0.233 & 4 & 0 & 0 & 8 & 8 \\
\hline \multirow{4}{*}{$\begin{array}{l}\text { Perceived } \\
\text { Playfulness }\end{array}$} & \multirow[t]{4}{*}{0.160} & $F 41$ & 0.028 & 2 & 4 & 4 & 8 & 2 \\
\hline & & $F+2$ & 0.258 & 4 & 8 & 4 & 4 & 0 \\
\hline & & F43 & 0.238 & 0 & 16 & 0 & 4 & 0 \\
\hline & & $F+4$ & 0.218 & 0 & 14 & 4 & 2 & 0 \\
\hline
\end{tabular}
consistency. In the same way, weights of other criteria (i.e., cost, perceived playfulness, perceived risk) can be calculated and depicted in table 2 .

Table 2.Criteria weight and evaluation 


\section{Analysis of MC applications accepted possibility using fuzzy comprehensive evaluation method}

\subsection{Fuzzy comprehensive evaluation model}

The comprehensive fuzzy evaluation model proposed is based on fuzzy set theory as developed by Zadeh and the analytic hierarchical process developed by Saaty. Zadeh define fuzzy logic underlying models of reasoning which are approximate rather exact. Saaty advocated the use of deductive systems approach in the analysis of complex decision problem. The steps in CFEM are described as follows.

1. Define model input, the set of judgment factors $\mathcal{U}_{i}(\boldsymbol{i}=1,2, \ldots, m)$, for the comprehensive fuzzy evaluation model. $U=\left\{u_{1}, u_{2}, u_{3}, \ldots, u_{m}\right\}, U$ is set of judgment factors.

2. Set the linguistic variable, $V=\left\{y_{1}, y_{2}, y_{3}, \ldots, y_{n}\right\}$ (comment factor)

Linguistic value $v_{i}(i=1,2, \ldots, n)$ is the result of evaluation, namely $V=($ Very Poor, Poor, Average, Good, Very Good $)$. Then give the value by five level measurements, $C=\left(\begin{array}{lllll}1 & 2 & 3 & 4 & 5\end{array}\right)$

3. Define the weightings of the judgment factors, $u_{i}(i=1,2, \ldots, m), W$ is corresponded fuzzy weightings vector, $W=\left(w_{1}, w_{2}, \ldots, w_{m}\right)$, based on questionnaire results.

4. Constructs the membership function $r(v)$. Membership function is at the core of fuzzy models. The membership function is considered to be the strongest and weakest point of fuzzy set theory. Member functions are the most commonly used.

5. Computer the degree of membership ( $\left.r_{i j}\right)$ and the fuzzy matrix $R, R$ is $R=\left(r_{i}, r_{i,}, r_{i}, \ldots, r_{n}\right)$

The fuzzy matrix is

$$
R=\left(\begin{array}{ccccc}
r_{11} & r_{12} & r_{13} & \ldots & r_{1 n} \\
r_{21} & r_{22} & r_{23} & \ldots & r_{2 s} \\
\ldots & \ldots & \ldots & \ldots & \ldots \\
r_{m 1} & r_{m 2} & r_{m 3} & \ldots & r_{m n}
\end{array}\right)
$$

6. Define the fuzzy judgment $B=W \cdot R$. So as to assess the effects. $B$ is the fuzzy evaluation model, $b,(j=1,2, \ldots, n)$ is fuzzy evaluation index.

Comprehensive fuzzy evaluation model is $U=\left\{u_{1}, u_{2}, u_{3}, \ldots u_{m}\right\}$

\subsection{Empirical study}

\subsubsection{Classification of MC Applications}

There is potentially an unlimited number of MC applications (Varshney, 2001; Varshney \& Vetter, 2002), which leads to a demand for classification, since currently, it is almost impossible to cover the whole range of potential MC products and services (Lehner \&Watson, 2001). Yuan and Zhang (2003) argue that value propositions in $\mathrm{MC}$ which define the relationship between seller offerings and buyer 
purchases by identifying how the seller achieves the buyer's needs (Clarke, 2001) originate from mobility and location awareness and are contrary to Internet-based ecommerce. Therefore, they group various MC applications based on these value propositions into six categories, namely ubiquitous communication, Emergency and time critical information services, location-sensitive service, pocket e-wallet, portable entertainment, improving productivity of mobile workforce. In this study, we adopt this classification and take example for ubiquitous communication.

\subsubsection{Construct evaluating criteria system}

The criteria system is depicted as figure 2 . in second section.

\subsubsection{Evaluate weight of criteria}

The process of evaluating weight of criteria can see from third section, and result is showed in table 2.

\subsubsection{Establish judgment matrix}

We investigate 20 consumers by questionnaire and let them objective score according to their own perception. The result is showed in table 2.The number of perception in table (i.e., very poor, poor, average, good, very good) represent how many consumers choose the answer.

$$
r_{i j}=\frac{c_{i j}}{\sum_{i=1}^{5} c_{i}}(i=1,2,3,4,)
$$

$\sum^{5} c_{i}=20$ is the total number of investigated consumers. According to the formula, we $i=\mathrm{d}$ obtain criteria judgment matrix:

$$
R_{1}=\left(\begin{array}{ccccc}
0.2 & 0 & 0.2 & 0.55 & 0.05 \\
0.05 & 0.05 & 0.15 & 0.3 & 0.45 \\
0 & 0 & 0.4 & 0.6 & 0 \\
0 & 0.15 & 0.4 & 0.45 & 0
\end{array}\right)
$$

\subsubsection{Fuzzy comprehensive evaluation aiming at objective}

$$
\begin{aligned}
& B_{1}=W_{1} \cdot R_{1}=\left(\begin{array}{lllll}
0.079 & 0.0381 & 0.2442 & 0.45095 & 0.18775
\end{array}\right) \\
& \text { In the same way, } \\
& B_{2}=\left(\begin{array}{llllll}
0.05078 & 0.12149 & 0.26474 & 0.32727 & 0.23125
\end{array}\right) \\
& B_{3}=\left(\begin{array}{lllll}
0.19694 & 0.04962 & 0.07672 & 0.24808 & 0.42864
\end{array}\right) \\
& B_{4}=\left(\begin{array}{lllll}
0.08025 & 0.52321 & 0.15242 & 0.23649 & 0.02863
\end{array}\right)
\end{aligned}
$$

And then, $B=W \cdot R=\left(\begin{array}{lllll}0.09761 & 0.14459 & 0.19534 & 0.32416 & 0.23195\end{array}\right)$ 


\subsubsection{Calculate accepted possibility}

$$
\begin{aligned}
& A D=B \cdot C^{T}=3.4292 \\
& A D P=3.4292 / 5 \cdot 100 \%=68.58 \%
\end{aligned}
$$

Therefore, as a whole, accepted possibility of this $\mathrm{MC}$ applications (i.e., ubiquitous communication is $68.58 \%$.

\section{Conclusions and future work}

This research has contributed to the MC field by exploring which factors affect consumer acceptance and $\mathrm{MC}$ application acceptance possibility by empirical study.

Some suggestions for future study are outlined here: first, TAM needs to be given additional variables to provide an even stronger model. Although some new factors are already introduced into TAM, for example research by Venkatesh and Davis indicated that both social influence processes and cognitive instrumental processes significantly influenced user, more reasonable variables is necessary to adopt to study consumer mc applications acceptance. Second, other research methodologies can be applied to study mc acceptance in order to do further research with a large population besides TAM. Third, empirical study should be further developed, including questionnaire design, choosing research object, scope of research etc. in this way, we can draw precise conclusion to guide mc healthy development.

\section{References}

1. B. Anckar and D. Dincau, "Value Creation in Mobile Commerce: Findings from a Consumer Survey", Journal of Information Technology Theory and Application. 4(1), 43-64(2002).

2. F. D. Davis, "Perceived Usefulness, Perceived Ease of Use, and User Acceptance of Information Technology", MIS Quarterly. 13(3), 319-340(1989).

3. B. Szajna, "Empirical Evaluation of the Revised Technology Acceptance Model", Management Science. 42(1), 85-92(1996).

4. J. H. Wu and S. C. Wang, "What Drives Mobile Commerce? An Empirical Evaluation of the Revised Technology Acceptance Model", Information Management. 42, 719-729(2005).

5. P.A. Pavlou, "What Drives Electronic Commerce? A Theory of Planned Behavior Perspective"( 2001); http://www-scf.usc.edu/pavlou/14794.pdf.

6. M. A. Atkinson and C. Kydd, "Individual Characteristics Associated with World Wide Web Use: An Empirical Study of Playfulness and Motivation", Data Base for Advances in Information Systems. 28(2), 53-62(1997).

7. T. Fenech, "Using Perceived Ease of Use and Perceived Usefulness to Predict Acceptance of the World Wide Web", Computer Network and ISDN Systems.30, 629-630(1998) 